\title{
The comeback kid: TYSABRI now FDA approved for Crohn disease
}

$T_{1}$ he US FDA recently approved the monoclonal antibody TYSABRI (natalizumab) for use in the treatment of adults with moderately to severely active Crohn disease $(\mathrm{CD})$ who do not respond to, or do not tolerate, conventional therapies for $\mathrm{CD}$ and inhibitors of TNF- $\alpha$. This represents a comeback for a drug that was temporarily pulled from the market in February 2005.

TYSABRI is a monoclonal antibody specific for the $\alpha_{4}$ integrin that pairs with either the $\beta_{1}$ integrin or $\beta_{7}$ integrin to form $\alpha_{4} \beta_{1}$ (also known as VLA4) and $\alpha_{4} \beta_{7}$, respectively. These integrin heterodimers are expressed by distinct subsets of $\mathrm{T}$ cells, and binding of $\alpha_{4} \beta_{1}$ and $\alpha_{4} \beta_{7}$ to their respective ligands is important for directing $\mathrm{T}$ cell migration to different tissues. Interactions between $\alpha_{4} \beta_{7}$ and MAdCAM-1 direct $\mathrm{T}$ cells to the intestine, both under steady-state conditions and during inflammation, whereas interactions between $\alpha_{4} \beta_{7}$ and VCAM- 1 are important for directing $\mathrm{T}$ cells to the inflamed CNS. Given its specificity for the $\alpha_{4}$ integrin, TYSABRI blocks $\mathrm{T}$ cell homing to the intestine and the inflamed CNS.

Two randomized, placebo-controlled phase III clinical trials indicated that TYSABRI markedly reduced the number of relapses in individuals with MS (1). The results were so promising that the FDA approved TYSABRI for the treatment of individuals with relapsing forms of MS in November 2004, only halfway through the phase III clinical trials. However, Elan Corp. and Biogen Idec, the manufacturers of TYSABRI, pulled the drug from the market and halted all ongoing clinical trials only 3 months later, after two patients developed progressive multifocal leukoencephalopathy (PML) - a demyelinating brain disorder caused by a polyomavirus known as the JC virus. Although one patient was successfully treated for the disorder (2), the other died. An additional fatal case of PML was uncovered when the data from one of the halted clinical trials, which had been assessing the efficacy of TYSABRI as a treatment for $\mathrm{CD}$, was retrospectively reviewed (3).

A review of the data from all clinical trials (i.e., the completed and halted trials assessing the efficacy of TYSABRI in patients with MS as well as the halted trials assessing the efficacy of the drug in patients with $C D$ ) revealed no more cases of PML. The FDA therefore reapproved the use of TYSABRI to treat individuals with relapsing forms of MS in June 2005 (4). However, because the two affected patients with MS

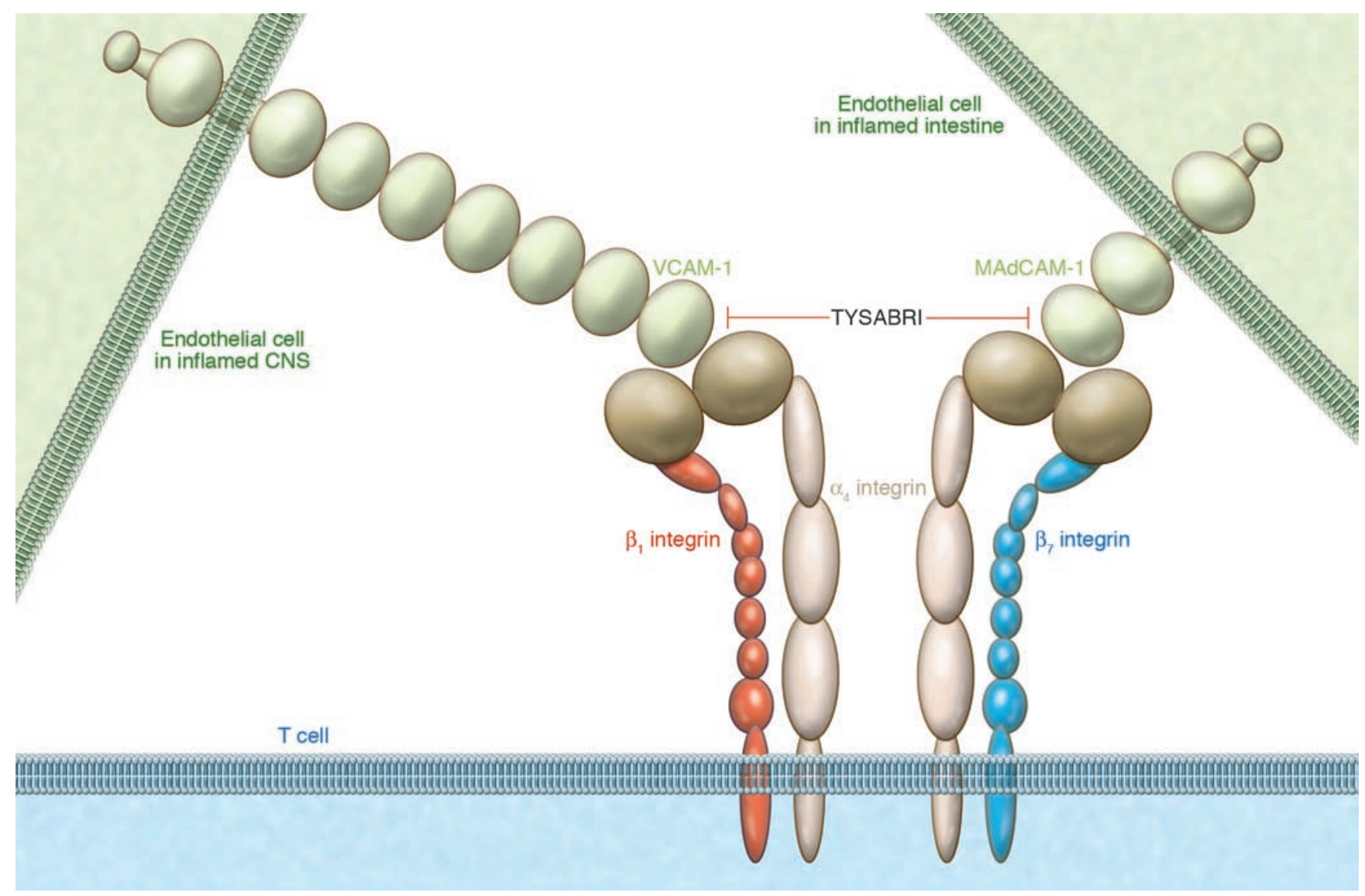

TYSABRI is a monoclonal antibody specific for the $\alpha_{4}$ integrin. It mediates its disease-modifying effects in individuals with CD and MS by preventing T cell recruitment to the inflamed intestine and inflamed CNS, tissues that express ligands for $\alpha_{4} \beta_{1}$ and $\alpha_{4} \beta_{7}$, respectively. 
were also being treated with IFN- $\beta$ and the affected patient with CD had a history of being treated with immunosuppressive drugs, the FDA restricted the use of TYSABRI such that even today, it can only be given to individuals with relapsing forms of MS who have not responded adequately to, or cannot tolerate, other treatments for MS, and it must be given as a monotherapy. Furthermore, TYSABRI remains available in the US only through a risk management program developed by the manufacturers of TYSABRI in conjunction with the FDA known as the TOUCH (TYSABRI Outreach: Unified Commitment to Health) prescribing program. In the European Union, where TYSABRI was approved for the treatment of individuals with relapsing forms of MS in June 2006, there is no restrictive prescribing program.

The TOUCH prescribing program facilitates the appropriate use of TYSABRI and ensures that patients are monitored very closely for any new sign or symptom suggestive of PML. Under the program, TYSABRI can only be prescribed, distributed, and infused by prescribers, infusion centers, and pharmacies associated with infusion centers registered with the program. Furthermore, patients receiving the drug must be educated about the risks and benefits of TYSABRI and enrolled in the TOUCH prescribing program.

Through the TOUCH prescribing program, more than 12,000 individuals with MS are being treated with TYSABRI. A similar CD-TOUCH prescribing program will be the only way that individuals with $\mathrm{CD}$ who fit all the criteria for receiving treatment with TYSABRI can receive the drug. Implementing this restricted distribution program for the network of physicians, nurses, and patients with $\mathrm{CD}$ has meant that although the drug is already FDA approved, as the JCI went to press, Elan Corp. and Biogen Idec anticipated that TYSABRI was likely to be available to individuals with CD only by the end of February 2008 (5).

It is estimated that there are 500,000 individuals in the US with CD, a substantial proportion of whom either fail or cannot tolerate current therapies. In clinical trials, TYSABRI has been shown to induce and maintain disease remission in individuals with moderate to severe $\mathrm{CD}$ for longer than two years (6). Therefore, as Stephen Hanauer, chief of the Section of Gastroenterology at the University of Chicago, said, "The FDA's approval of TYSABRI is an important step forward in the treatment of Crohn's disease," because, as he explained, "The unique mechanism of action of TYSABRI affords us a new class of therapy in our fight against this debilitating disease" (5).

\section{Karen Honey}

1. Polman, C.H., et al. 2006. A randomized, placebocontrolled trial of natalizumab for relapsing multiple sclerosis. N. Engl. J. Med. 354:899-910.

2. Langer-Gould, A., Atlas, S.W., Green, A.J., Bollen, A.W., and Pelletier, D. 2005. Progressive multifocal leukoencephalopathy in a patient treated with natalizumab. N. Engl. J. Med. 353:375-381.

3. Van Assche, G., et al. 2005. Progressive multifocal leukoencephalopathy after natalizumab therapy for Crohn's disease. N. Engl. J. Med. 353:362-368.

4. US FDA. 2005. Natalizumab (marketed as Tysabri) information. http://www.fda.gov/cder/drug/ infopage/natalizumab/default.htm.

5. Biogen Idec news and media. http://www.biogenidec.com/site/news-and-media.html.

6. Elan Pharmaceuticals Inc. 2006. TYSABRI(R) maintained remission in patients with moderate-to-severe Crohn's disease treated for longer than two years according to data presented this week. October 24, 2006. http://www.elan.com/ News/2006/20061024.asp. 